\title{
Model Reduction for Controllable Systems
}

\author{
Ha B.M., P. Rapisarda, and H.L. Trentelman
}

\begin{abstract}
In the papers [1], [7] a new scheme for passivitypreserving model reduction has been proposed. We have shown in [2] that the approach can also be interpreted from a dissipativity theory point of view, and we put forward two procedures in order to compute a driving variable or output nulling representation of a reduced order model for a given behavior. In this paper we illustrate improved versions of both algorithms, which produce a controllable reduced-order model. The new algorithms are based on several original results of independent interest.
\end{abstract}

\section{INTRODUCTION}

Recently, Antoulas (see [1]) and Sorensen (see [7]) have presented a new technique and efficient numerical algorithms in order to perform model reduction with passivity- and stability preservation. In [2] we offered a different point of view on their approach, using ideas from the behavioral theory of dissipative systems, and we cast the methods of Antoulas and Sorensen in a general framework for model reduction, applicable also when the original system is not passive. In our approach, one is given a system $\mathfrak{B}$ of McMillan degree $\mathrm{n}$ which is half-line dissipative with respect to a given supply rate, and an integer $0<\mathrm{k}<\mathrm{n}$; the goal is to obtain a reduced-order model $\hat{\mathfrak{B}}$ of $\mathfrak{B}$, with McMillan degree less than or equal to $\mathrm{k}$, which is also halfline dissipative with respect to $\Sigma$.

In [2] we illustrated an algorithm to obtain a drivingvariable representation of the reduced-order model. The drawback of that procedure is that the reduced-order model is not guaranteed to be controllable, and consequently it is impossible to check its dissipativity. In this communication we present a new algorithm to compute a reduced-order model which is guaranteed to be controllable and dissipative. Moreover, we present a new procedure in order to compute an output-nulling representation of a reduced-order model.

Notation and background material. We denote by $\mathfrak{C}^{\infty}\left(\mathbb{R}, \mathbb{R}^{\mathrm{w}}\right)$ the set of infinitely often differentiable functions from $\mathbb{R}$ to $\mathbb{R}^{w}$, with $\mathfrak{D}\left(\mathbb{R}, \mathbb{R}^{w}\right)$ the subspace of $\mathfrak{C}^{\infty}\left(\mathbb{R}, \mathbb{R}^{\mathrm{w}}\right)$ consisting of all compactly supported functions, with $\mathfrak{L}_{2}^{\text {loc }}\left(\mathbb{R}, \mathbb{R}^{\mathrm{w}}\right)$ the set of all Lebesgue measurable functions $w$ from $\mathbb{R}$ to $\mathbb{R}^{w}$ for which the integral $\int_{\Omega}\|w\|^{2} d t$ is finite for all compact sets $\Omega \subset \mathbb{R}$.

A subset $\mathfrak{B} \subset \mathfrak{L}_{2}^{\text {loc }}\left(\mathbb{R}, \mathbb{R}^{\mathrm{w}}\right)$ defines a linear differential system if there exists a polynomial matrix $R \in \mathbb{R}^{\mathrm{w} \times \mathrm{w}}[\xi]$ such that $\mathfrak{B}=\left\{w \in \mathfrak{L}_{2}^{\text {loc }}\left(\mathbb{R}, \mathbb{R}^{\mathrm{w}}\right) \mid R(d / d t) w=0\right\}$. We denote with $\mathfrak{L}^{\mathrm{w}}$ the set of linear differential systems with w external variables.

We call $\mathfrak{B} \in \mathfrak{L}^{\mathrm{w}}$ controllable if for all $w_{1}, w_{2} \in \mathfrak{B}$, there exists a $T \geq 0$ and a $w \in \mathfrak{B}$ such that $w(t)=w_{1}(t)$ for $t<0$ and $w(t+\bar{T})=w_{2}(t)$ for $t \geq 0$. We denote the controllable elements of $\mathfrak{L}^{\mathrm{w}}$ by $\mathfrak{L}_{\text {contr }}^{\mathrm{w}}$. The controllable part of a behavior is defined as follows. Let $\mathfrak{B} \in \mathfrak{L}^{\text {w }}$. It can be shown that there exists $\mathfrak{B}^{\prime} \in \mathfrak{L}_{\text {contr }}^{\mathrm{w}}, \mathfrak{B}^{\prime} \subset \mathfrak{B}$ such that $\mathfrak{B}^{\prime \prime} \in \mathfrak{L}_{\text {contr }}^{\mathrm{w}}, \mathfrak{B}^{\prime \prime} \subset \mathfrak{B}$ implies $\mathfrak{B}^{\prime \prime} \subset \mathfrak{B}^{\prime}$, i.e, $\mathfrak{B}^{\prime}$ is the largest controllable subbehavior contained in $\mathfrak{B}$. Denote this system as $\mathfrak{B}_{\text {contr. }}$.

There are a number of important integer invariants associated with behaviors. The integer invariants associated with a linear differential behavior $\mathfrak{B}$ are the number of inputs, denoted $\mathrm{m}(\mathfrak{B})$, the number of outputs, denoted $\mathrm{p}(\mathfrak{B})$, and the dimension of a minimal state variable for $\mathfrak{B}$, equivalently called the McMillan degree of $\mathfrak{B}$ and denoted with $\mathrm{n}(\mathfrak{B})$.

Given a controllable linear differential behavior $\mathfrak{B} \in \mathfrak{L}_{\text {cont }}^{\mathrm{w}}$ and $\Sigma=\Sigma^{\top} \in \mathbb{R}^{\mathrm{w} \times \mathrm{w}}$ nonsingular, we define its $\Sigma$ orthogonal complement $\mathfrak{B}^{\perp_{\Sigma}}$ as

$$
\begin{array}{r}
\mathfrak{B}^{\perp_{\Sigma}}:=\left\{w \in \mathfrak{L}_{2}^{l o c}\left(\mathbb{R}, \mathbb{R}^{\mathrm{w}}\right) \mid \int_{-\infty}^{+\infty} w^{\top} \Sigma \Delta \mathrm{d} t=0\right. \\
\text { for all } \left.\Delta \in \mathfrak{B} \cap \mathfrak{D}\left(\mathbb{R}, \mathbb{R}^{\mathrm{w}}\right)\right\} .
\end{array}
$$

The $\Sigma$-orthogonal complement $\mathfrak{B}^{\perp_{\Sigma}}$ is again an element of $\mathfrak{L}^{\mathrm{w}}$, and it is controllable, see section 10 of [11]. When $\Sigma=$ $I$, we simply write $\mathfrak{B}^{\perp}$ and call it the orthogonal complement of $\mathfrak{B}$.

\section{STATIONARY TRAJECTORIES AND DISSIPATIVE SYSTEMS}

The notion of stationarity of a trajectory and that of dissipativity of a system will play an important role in the following, and we briefly review them now.

Definition 1: Let $\mathfrak{B} \in \mathfrak{L}_{\text {contr }}^{\mathrm{w}}$, and $\Sigma=\Sigma^{\top} \in \mathbb{R}^{\mathrm{w} \times \mathrm{w}}$ be nonsingular. We call $w \in \mathfrak{B}$ a stationary trajectory with respect to $\Sigma$ if the linear term in the variation $\Delta \in \mathfrak{B} \cap$ $\mathfrak{D}\left(\mathbb{R}, \mathbb{R}^{\mathrm{w}}\right)$ in the integral

$$
\int_{-\infty}^{+\infty}\left[(w+\Delta)^{\top} \Sigma(w+\Delta)-w^{\top} \Sigma w\right] \mathrm{d} t
$$

is the zero functional.

We denote the subset of stationary trajectories of $\mathfrak{B}$ with respect to $\Sigma$ with the symbol $\mathfrak{B}^{*}$.

Integrating by parts the integral appearing in Definition 1 it can be verified that the linear term equals

$$
2 \int_{-\infty}^{+\infty} w^{\top} \Sigma \Delta \mathrm{d} t
$$

Consequently, the set of stationary trajectories of $\mathfrak{B}$ with respect to $\Sigma$ is

$$
\begin{aligned}
\mathfrak{B}^{*} & =\left\{w \in \mathfrak{B} \mid \int_{-\infty}^{+\infty} w^{\top} \Sigma \Delta \mathrm{d} t=0\right. \\
& \text { for all } \left.\Delta \in \mathfrak{B} \cap \mathfrak{D}\left(\mathbb{R}, \mathbb{R}^{\mathrm{w}}\right)\right\} \\
& =\mathfrak{B} \cap \mathfrak{B}^{\perp_{\Sigma}} .
\end{aligned}
$$


This leads to the following characterization of $\mathfrak{B}^{*}$, which relates the concept of stationarity with the notion of duality. For a proof, see [6].

Proposition 2: Let $\mathfrak{B} \in \mathfrak{L}_{\text {contr }}^{\mathrm{w}}$ and let $\Sigma=\Sigma^{\top} \in \mathbb{R}^{\mathrm{w} \times \mathrm{w}}$ be nonsingular. Then $\mathfrak{B}^{*} \in \mathfrak{L}^{\mathrm{w}}$, and is given by

$$
\mathfrak{B}^{*}=\mathfrak{B} \cap \mathfrak{B}^{\perp_{\Sigma}}=\mathfrak{B} \cap[\Sigma \mathfrak{B}]^{\perp} .
$$

We now give the definition of (strict-) dissipativity; for a through treatment of the concept of dissipativity and its consequences see [11].

Definition 3: Let $\mathfrak{B} \in \mathfrak{L}_{\text {contr }}^{\mathrm{w}}$ and let $\Sigma=\Sigma^{\top} \in \mathbb{R}^{\mathrm{w} \times \mathrm{w}}$ be nonsingular.

1) $\mathfrak{B}$ is $\Sigma$ - dissipative if and only if $\int_{\mathbb{R}} w^{\top} \Sigma w d t \geq 0$ for all $w \in \mathfrak{B} \cap \mathfrak{D}\left(\mathbb{R}, \mathbb{R}^{w}\right)$;

2) $\mathfrak{B}$ is strictly $\Sigma$-dissipative if and only if there exists $\varepsilon_{0}>0$ such that $\int_{\mathbb{R}} w^{\top} \Sigma w d t \geq \varepsilon_{0} \int_{\mathbb{R}} w^{\top} w d t$ for all $w \in \mathfrak{B} \cap \mathfrak{D}\left(\mathbb{R}, \mathbb{R}^{w}\right)$;

3) $\mathfrak{B}$ is strictly $\Sigma$-dissipative on $\mathbb{R}_{-}$if there exists $\varepsilon_{0}>0$ such that $\int_{\mathbb{R}_{-}} w^{\top} \Sigma w d t \geq \varepsilon_{0} \int_{\mathbb{R}_{-}} w^{\top} w d t$ for all $w \in$ $\mathfrak{B} \cap \mathfrak{D}\left(\mathbb{R}_{-}, \mathbb{R}^{\mathrm{w}}\right)$;

4) $\mathfrak{B}$ is strictly $\Sigma$-dissipative on $\mathbb{R}_{+}$if there exists $\varepsilon_{0}>0$ such that $\int_{\mathbb{R}_{+}} w^{\top} \Sigma w d t \geq \varepsilon_{0} \int_{\mathbb{R}_{+}} w^{\top} w d t$ for all $w \in$ $\mathfrak{B} \cap \mathfrak{D}\left(\mathbb{R}_{+}, \mathbb{R}^{\mathrm{w}}\right)$;

Finally, we consider the consequences of strict half-line dissipativity of $\mathfrak{B}$ on the set of stationary trajectories $\mathfrak{B}^{\text {ast }}$.

Proposition 4: Let $\mathfrak{B} \in \mathfrak{L}_{\text {contr }}^{\mathrm{w}}$ and let $\Sigma=\Sigma^{\top} \in \mathbb{R}^{\mathrm{w} \times \mathrm{w}}$ be nonsingular. Assume that $\mathfrak{B}$ is strictly $\Sigma$-dissipative on $\mathbb{R}_{-}$ (or $\mathbb{R}_{+}$), then

1) $\mathfrak{B}^{*}$ coincides with the set of locally minimal trajectories, i.e. for $w \in \mathfrak{B}^{*}$

$$
\int_{-\infty}^{+\infty}\left[(w+\Delta)^{\top} \Sigma(w+\Delta)-w^{\top} \Sigma w\right] \mathrm{d} t \geq 0
$$

for all $\Delta \in \mathfrak{B} \cap \mathfrak{D}\left(\mathbb{R}, \mathbb{R}^{w}\right)$;

2) $\mathfrak{B}^{*}$ is an autonomous behavior;

3) $\mathrm{n}\left(\mathfrak{B}^{*}\right)=2 \mathrm{n}(\mathfrak{B})$.

\section{PROBLEM FORMULATION}

In this paper we illustrate procedures in order to solve the following problem. Problem Let $\mathfrak{B} \in \mathfrak{L}_{\text {contr }}^{\mathrm{w}}$ be strictly halfline dissipative on $\mathbb{R}^{-}$with respect to $\Sigma$, with $\Sigma=\Sigma^{\top} \in$ $\mathbb{R}^{\mathrm{W} \times \mathrm{w}}$ nonsingular. Let $\mathrm{k}<\mathrm{n}(\mathfrak{B})$ be given together with a subbehavior $\mathfrak{B}^{\prime} \subset\left[\mathfrak{B}^{*}\right]_{\text {anistable }}$ such that $\mathrm{n}\left(\mathfrak{B}^{\prime}\right)=\mathrm{k}$, where $\left[\mathfrak{B}^{*}\right]_{\text {antistable }}$ is the anti-stable part of $\mathfrak{B}^{*}$. Find $\hat{\mathfrak{B}} \in \mathfrak{L}_{\text {contr }}^{\mathrm{w}}$ such that

1) $\mathrm{n}(\hat{\mathfrak{B}}) \leq \mathrm{k}$

2) $\hat{\mathfrak{B}}$ is strictly dissipative on $\mathbb{R}^{-}$with respect to $\Sigma$;

3) The anti-stable part $\left[\hat{\mathfrak{B}}^{*}\right]_{\text {antistable }}$ of $\hat{\mathfrak{B}}^{*}$ is a subbehavior of $\mathfrak{B}^{\prime}$.

In the next sections we will solve this problem and compute a driving-variable representation and output-nulling representation of the reduced order behavior $\hat{\mathfrak{B}}$.

\section{DRIVING VARIABLE REPRESENTATIONS}

Let $A \in \mathbb{R}^{\mathrm{n} \times \mathrm{n}}, B \in \mathbb{R}^{\mathrm{n} \times \mathrm{m}}, C \in \mathbb{R}^{\mathrm{w} \times \mathrm{n}}, D \in \mathbb{R}^{\mathrm{w} \times \mathrm{m}}$ be constant real matrices. The equations

$$
\dot{x}=A x+B v, w=C x+D v .
$$

represent the behavior

$$
\mathfrak{B}_{D V}(A, B, C, D):=\{(w, x, v) \mid(1) \text { hold }\} .
$$

This behavior is called the full behavior represented by (1). If we eliminate $x$ and $v$, then we get the external behavior defined by

$$
\begin{aligned}
\mathfrak{B}_{D V}(A, B, C, D)_{\text {ext }}:= & \{w \mid \exists x, v \text { such that } \\
& \left.(w, x, v) \in \mathfrak{B}_{D V}(A, B, C, D)\right\} .
\end{aligned}
$$

It is well-known that for any given $\mathfrak{B} \in \mathfrak{L}^{\mathrm{w}}$ there exist real constant matrices $A, B, C, D$ such that (see [8])

$$
\mathfrak{B}=\mathfrak{B}_{D V}(A, B, C, D)_{\text {ext }} .
$$

In this case we call $\mathfrak{B}_{D V}(A, B, C, D)$ a driving variable representation of $\mathfrak{B}$. If $\mathrm{n}$ and $\mathrm{m}$ are minimal over all such driving variable representations, then we call $\mathfrak{B}_{D V}(A, B, C, D)$ a minimal driving variable representation. $\mathfrak{B}_{D V}(A, B, C, D)_{\text {ext }}$ can be shown to be controllable if and only if the pair $(A, B)$ is controllable.

If a behavior is strictly-dissipative, then there exists a driving variable representation with some special properties.

Proposition 5: Let $\mathfrak{B} \in \mathfrak{L}_{\text {contr }}^{\mathrm{w}}$ be strictly $\Sigma$-dissipative, $\Sigma=\Sigma^{\top} \in \mathbb{R}^{\mathrm{w} \times \mathrm{w}}$ be nonsingular. Then, there exist constant matrices $A, B, C, D$ such that $\mathfrak{B}_{D V}(A, B, C, D)$ is a minimal driving variable representation of $\mathfrak{B}$, with

1) $(A, B)$ is controllable.

2) $D^{\top} \Sigma D=I$.

3) $D^{\top} \Sigma C=0$.

Hence, for sake of simplicity and without loss of generality, in the rest of this paper we make the following assumptions.

Assumption 1. $\mathfrak{B}_{D V}(A, B, C, D)$ is minimal.

Assumption 2. The pair $(A, B)$ is controllable.

Assumption 3. $D^{\top} \Sigma D=I$.

Assumption 4. $D^{\top} \Sigma C=0$.

\section{A. Characterization of dissipative DV representations}

We now characterize the dissipativity of systems represented in driving variable representation and find a way to compute the stationary trajectories of these systems.

Proposition 6: Let Assumptions 1, 2, 3, 4 hold. Then the following conditions are equivalent:

1. $\mathfrak{B}$ is strictly dissipative on $\mathbb{R}_{-}$with respect to $\Sigma$.

2. The ARE

$$
A^{\top} K+K A+K B B^{\top} K-C^{\top} \Sigma C=0 ;
$$

has unique solution $X$ such that:

a) $K>0$; and

b) $A+B B^{\top} K$ is antistable;

Under the same assumptions, the two following conditions are equivalent:

3. $\mathfrak{B}$ is strictly dissipative on $\mathbb{R}_{+}$with respect to $\Sigma$.

4. The ARE

$$
A^{\top} K+K A+K B B^{\top} K-C^{\top} \Sigma C=0 ;
$$


has unique solution $K$ such that:

a) $K<0$; and

b) $A+B B^{\top} K$ is stable;

\section{B. Stationary trajectories of driving variable representations}

Let $\mathfrak{B} \in \mathfrak{L}_{\text {contr }}^{\mathrm{w}}$ be $\Sigma$-dissipative, $\Sigma=\Sigma^{\top} \in \mathbb{R}^{\mathrm{w} \times \mathrm{w}}$ be nonsingular. Let $\mathfrak{B}_{D V}(A, B, C, D)$ be a driving variable representation of $\mathfrak{B}$.

In order to compute the stationary trajectories of $\mathfrak{B}$ in terms of the driving variable representation, we use the result of Proposition 2. It can be shown that if $\mathfrak{B}_{D V}(A, B, C, D)$ is a minimal driving variable representation of a controllable behavior $\mathfrak{B}$, then (see [12]) $\mathfrak{B}_{O N}\left(-A^{\top}, C^{\top} \Sigma, B^{\top},-D^{\top} \Sigma\right)$ is minimal output nulling representation of $\mathfrak{B}^{\perp_{\Sigma}}$ (see Section $\mathrm{V}$ for a definition of output nulling representation). Consequently, the set of stationary trajectories of $\mathfrak{B}$ can be represented as follows:

$$
\begin{aligned}
\mathfrak{B}^{*}= & \mathfrak{B}_{D V}(A, B, C, D)_{\text {ext }} \\
& \cap \mathfrak{B}_{O N}\left(-A^{\top}, C^{\top} \Sigma, B^{\top},-D^{\top} \Sigma\right)_{\text {ext }} .
\end{aligned}
$$

We define

$$
\begin{gathered}
\mathfrak{B}_{H}(A, B, C, D):=\mathfrak{B}_{D V}(A, B, C, D)_{e x t} \\
\cap \mathfrak{B}_{O N}\left(-A^{\top}, C^{\top} \Sigma, B^{\top},-D^{\top} \Sigma\right)_{e x t}
\end{gathered}
$$

and we call it the Hamiltonian subbehavior of $\mathfrak{B}$. Indeed, if assumptions 3,4 hold then $\mathfrak{B}_{H}(A, B, C, D)$ is the autonomous behavior generated by the Hamiltonian matrix, as the following result shows.

Proposition 7: Let Assumptions 3, 4 hold. Then $\mathfrak{B}_{H}(A, B, C, D)$ consists of those $w \in \mathfrak{L}_{2}^{\text {loc }}\left(\mathbb{R}, \mathbb{R}^{\mathrm{w}}\right)$ for which exist $x, z \in \mathfrak{L}_{2}^{\text {loc }}\left(\mathbb{R}, \mathbb{R}^{\mathrm{n}}\right)$ such that

$$
\begin{aligned}
{\left[\begin{array}{c}
\dot{x} \\
\dot{z}
\end{array}\right] } & =\left[\begin{array}{cc}
A & B B^{\top} \\
C^{\top} \Sigma C & -A^{\top}
\end{array}\right]\left[\begin{array}{l}
x \\
z
\end{array}\right] \\
w & =\left[\begin{array}{ll}
C & D B^{\top}
\end{array}\right]\left[\begin{array}{c}
x \\
z
\end{array}\right] .
\end{aligned}
$$

The following result shows that we can use the Hamiltonian subbehavior of $\mathfrak{B}$, in order compute the antistable part of the set stationary trajectories.

Proposition 8: Let $\mathfrak{B} \in \mathfrak{L}_{\text {contr }}^{\mathrm{w}}, \quad \Sigma=\Sigma^{\top} \in \mathbb{R}^{\mathrm{w} \times \mathrm{w}}$ be nonsingular. Let $\mathfrak{B}_{D V}(A, B, C, D)$ be a driving variable representation of $\mathfrak{B}$ and satisfy assumption $1,2,3,4$. Then

1. $\mathfrak{B}^{*}$ is the external behavior of $\mathfrak{B}_{H}(A, B, C, D)$ given in (4).

2. $\left[\mathfrak{B}^{*}\right]_{\text {antistable }}=\operatorname{span}\left\{C e^{\Lambda_{u} t} X_{1}+D B^{\top} e^{\Lambda_{u} t} Y_{1}\right\}$, where $X_{1} \in \mathbb{R}^{\mathrm{n} \times \mathrm{n}}, Y_{1} \in \mathbb{R}^{\mathrm{n} \times \mathrm{n}}$ are such that $\operatorname{im}\left(\left[\begin{array}{c}X_{1} \\ Y_{1}\end{array}\right]\right)$ forms a basis for the set of right half-plane eigenvectors of $H$, i.e.

$$
\left[\begin{array}{cc}
A & B B^{\top} \\
C^{\top} \Sigma C & -A^{\top}
\end{array}\right]\left[\begin{array}{c}
X_{1} \\
Y_{1}
\end{array}\right]=\left[\begin{array}{c}
X_{1} \\
Y_{1}
\end{array}\right] \Lambda_{u},
$$

with $\sigma\left(\Lambda_{u}\right)=\left\{\lambda_{1}, \ldots, \lambda_{\mathrm{n}}\right\}, \lambda_{i} \in \sigma(H) \bigcap \mathbb{C}_{+}, i=$ $1, \ldots, \mathrm{n}$.

3. Let $\mathfrak{B}^{\prime} \subset\left[\mathfrak{B}^{*}\right]_{\text {antistable }}$ such that $\mathrm{n}\left(\mathfrak{B}^{\prime}\right)=\mathrm{k}$. Then there exist a permutation matrix $\Pi$ such that $X_{1} \Pi$ and $Y_{1} \Pi$ can be partitioned as $X_{1} \Pi=\left[\begin{array}{ll}X_{1}^{1} & X_{1}^{2}\end{array}\right]$ and $Y_{1} \Pi=$ $\left[Y_{1}^{1} Y_{1}^{2}\right]$ with $X_{1}^{1}$ and $Y_{1}^{1}$ having $\mathrm{k}$ columns, such that

$$
\begin{aligned}
& {\left[\begin{array}{cc}
A & B B^{\top} \\
C^{\top} \Sigma C & -A^{\top}
\end{array}\right]\left[\begin{array}{cc}
X_{1}^{1} & X_{1}^{2} \\
Y_{1}^{1} & Y_{1}^{2}
\end{array}\right]} \\
& =\left[\begin{array}{cc}
X_{1}^{1} & X_{1}^{2} \\
Y_{1}^{1} & Y_{1}^{2}
\end{array}\right] \underbrace{\left[\begin{array}{cc}
\Lambda_{11} & \Lambda_{12} \\
0 & \Lambda_{22}
\end{array}\right]}_{=: \Lambda_{u}},
\end{aligned}
$$

and $\mathfrak{B}^{\prime}=\operatorname{span}\left\{C e^{\Lambda_{11} t} X_{1}^{1}+D B^{\top} e^{\Lambda_{11} t} Y_{1}^{1}\right\}$, with $\sigma\left(\Lambda_{u}\right)=\left\{\lambda_{1}, \ldots, \lambda_{\mathrm{n}}\right\}$, where $\lambda_{i} \in \sigma(H) \bigcap \mathbb{C}_{+}$, $i=1, \ldots, \mathrm{n}$.

Next, we will find a representation of $\mathfrak{B}^{*}$ for the general, i.e. non-controllable case.

Proposition 9: Let $\mathfrak{B} \in \mathfrak{L}^{\mathrm{w}}, \Sigma=\Sigma^{\top} \in \mathbb{R}^{\mathrm{w} \times \mathrm{w}}$ be nonsingular. Let $\mathfrak{B}_{D V}(A, B, C, D)$ be a non necessarily controllable driving variable representation of $\mathfrak{B}$ and satisfy assumption 3 , 4. Then

1. $\left[\mathfrak{B}_{\text {contr }}\right]^{*} \subseteq \mathfrak{B}_{H}(A, B, C, D)$, where $\mathfrak{B}_{H}(A, B, C, D)$ is given in (4).

2. $\left[\mathfrak{B}_{\text {contr }}\right]_{\text {antistable }}^{*} \subseteq \operatorname{span}\left\{C e^{\Lambda_{u} t} X_{1}+D B^{\top} e^{\Lambda_{u} t} Y_{1}\right\}$, where $X_{1} \in \mathbb{R}^{\mathrm{n} \times \mathrm{n}}, Y_{1} \in \mathbb{R}^{\mathrm{n} \times \mathrm{n}}$ are such that $\operatorname{im}\left(\left[\begin{array}{c}X_{1} \\ Y_{1}\end{array}\right]\right)$ forms a basis for the set of right half-plane eigenvectors of $H$, i.e.

$$
\left[\begin{array}{cc}
A & B B^{\top} \\
C^{\top} \Sigma C & -A^{\top}
\end{array}\right]\left[\begin{array}{c}
X_{1} \\
Y_{1}
\end{array}\right]=\left[\begin{array}{c}
X_{1} \\
Y_{1}
\end{array}\right] \Lambda_{u}
$$

with $\sigma\left(\Lambda_{u}\right)=\left\{\lambda_{1}, \ldots, \lambda_{\mathrm{n}}\right\}, \lambda_{i} \in \sigma(H) \bigcap \mathbb{C}_{+}, i=$ $1, \ldots, \mathrm{n}$.

\section{OUTPUT NULLING REPRESENTATIONS}

Next, we talk about output nulling representations. Let $A \in \mathbb{R}^{\mathrm{n} \times \mathrm{n}}, B \in \mathbb{R}^{\mathrm{n} \times \mathrm{w}}, C \in \mathbb{R}^{\mathrm{p} \times \mathrm{n}}, D \in \mathbb{R}^{\mathrm{p} \times \mathrm{w}}$ be constant real matrices. The equations

$$
\dot{x}=A x+B w, \quad 0=C x+D w .
$$

represent the behavior

$$
\mathfrak{B}_{O N}(A, B, C, D):=\{(w, x) \mid(5) \text { hold }\} .
$$

This behavior is called the full behavior represented by (5). If we eliminate $x$, then we get the external behavior defined by

$$
\begin{aligned}
\mathfrak{B}_{O N}(A, B, C, D)_{\text {ext }}:= & \{w \mid \exists x \text { such that } \\
& \left.(w, x) \in \mathfrak{B}_{O N}(A, B, C, D)\right\} .
\end{aligned}
$$

It is well-known that for any given $\mathfrak{B} \in \mathfrak{L}^{\mathrm{w}}$ there exist real constant matrices $A, B, C, D$ such that (see [8])

$$
\mathfrak{B}=\mathfrak{B}_{O N}(A, B, C, D)_{\text {ext }} .
$$

In this case we call $\mathfrak{B}_{O N}(A, B, C, D)$ an output nulling representation of $\mathfrak{B}$, and if $\mathrm{n}$ and $\mathrm{p}$ are minimal over all such output nulling representations, then we call it a minimal one.

If $\mathfrak{B}$ is strictly dissipative, then without loss of generality we can make the following assumptions. 
Proposition 10: Let $\mathfrak{B} \in \mathfrak{L}_{\text {contr }}^{\mathrm{w}}$ be strictly $\Sigma$-dissipative, $\Sigma=\Sigma^{\top} \in \mathbb{R}^{\mathrm{w} \times \mathrm{w}}$ be nonsingular and $J=$ block $\operatorname{diag}\left(I_{\text {row (D)-q }},-I_{\mathrm{q}}\right)$, where $\mathrm{q}$ is number of negative eigenvalues of $\Sigma$. Then, there exist constant matrices $A, B, C, D$ such that $\mathfrak{B}_{O N}(A, B, C, D)$ is a minimal output nulling representation of $\mathfrak{B}$, with

1) $(A+F C, B+F D)$ controllable for all real matrices $F$.

2) $D \Sigma^{-1} D^{\top}=J$.

3) $B \Sigma^{-1} D^{\top}=0$.

Hence, for sake of simplicity and without loss of generality, we will use the following assumptions for our original output nulling representation.

Assumption 5. $\mathfrak{B}_{O N}(A, B, C, D)$ is a minimal representation of $\mathfrak{B}$.

Assumption 6. $(A+F C, B+F D)$ is controllable for all real matrices $F$.

Assumption 7. $D \Sigma^{-1} D^{\top}=J$.

Assumption 8. $B \Sigma^{-1} D^{\top}=0$.

In the following subsection we study how to characterize the dissipativity of systems represented in output nulling representation and how to compute the stationary trajectories of these systems.

\section{A. Characterization of dissipative ON representations}

Let $\mathfrak{B} \in \mathfrak{L}_{\text {contr }}^{\mathrm{w}}$, and consider an output nulling representation $\mathfrak{B}_{O N}(A, B, C, D)$ of $\mathfrak{B}$.

Proposition 11: Let Assumptions 5, 6, 7, 8 hold, $\Sigma=\Sigma^{\top} \in \mathbb{R}^{\mathrm{w} \times \mathrm{w}}$ be nonsingular and $J=$ block $\operatorname{diag}\left(I_{\text {row (D) }-\mathrm{q}},-I_{\mathrm{q}}\right)$, where $\mathrm{q}$ is number of negative eigenvalues of $\Sigma$. Then the two following conditions are equivalent:

1. $\mathfrak{B}$ is strictly dissipative on $\mathbb{R}_{-}$with respect to $\Sigma$.

2. The ARE

$$
A H+H A^{\top}-H C^{\top} J C H+B \Sigma^{-1} B^{\top}=0 ;
$$

has unique solution $H$ such that:

a) $H>0$; and

b) $A^{\top}-C^{\top} J C H$ is stable;

Similarly, the two following conditions are equivalent:

3. $\mathfrak{B}$ is strictly dissipative on $\mathbb{R}_{+}$with respect to $\Sigma$.

4. The ARE

$$
A H+H A^{\top}-H C^{\top} J C H+B \Sigma^{-1} B^{\top}=0 ;
$$

has unique solution $H$ such that:

a) $H<0$; and

b) $A^{\top}-C^{\top} J C H$ is antistable;

\section{B. Stationary trajectories of output nulling representations}

Let $\mathfrak{B} \in \mathfrak{L}_{\text {contr }}^{\mathrm{w}}$ be $\Sigma$-dissipative, $\Sigma=\Sigma^{\top} \in \mathbb{R}^{\mathrm{w} \times \mathrm{w}}$ be nonsingular and $J=\operatorname{block} \operatorname{diag}\left(I_{\text {row (D) }-\mathrm{q}},-I_{\mathrm{q}}\right)$, where $\mathrm{q}$ is number of negative eigenvalues of $\Sigma$. Let $\mathfrak{B}_{O N}(A, B, C, D)$ be a output nulling representation of $\mathfrak{B}$.

In order to compute the stationary trajectories of $\mathfrak{B}$ in terms of the output nulling representation we use the result of Proposition 2. It can be shown that if $\mathfrak{B}_{O N}(A, B, C, D)$ is a minimal output nulling representation of a controllable behavior $\mathfrak{B}$, then $\mathfrak{B}_{D V}\left(-A^{\top}, C^{\top}, B^{\top} \Sigma^{-1},-\Sigma^{-1} D^{\top}\right)$ is minimal output nulling representation of $\mathfrak{B}^{\perp_{\Sigma}}$. Hence, the set of stationary trajectories of the controllable system $\mathfrak{B}$ can be represented as

$$
\begin{aligned}
\mathfrak{B}^{*}= & \mathfrak{B}_{O N}(A, B, C, D)_{\text {ext }} \\
& \cap \mathfrak{B}_{D V}\left(-A^{\top}, C^{\top}, B^{\top} \Sigma^{-1},-\Sigma^{-1} D^{\top}\right)_{\text {ext }} .
\end{aligned}
$$

We define

$$
\begin{gathered}
\mathfrak{B}_{H^{\prime}}(A, B, C, D):=\mathfrak{B}_{O N}(A, B, C, D)_{\text {ext }} \\
\cap \mathfrak{B}_{D V}\left(-A^{\top}, C^{\top}, \Sigma^{-1} B^{\top},-\Sigma^{-1} D^{\top}\right)_{\text {ext }}
\end{gathered}
$$

and we call it the Hamiltonian subbehavior of $\mathfrak{B}$; indeed, if assumptions 7, 8 hold, then $\mathfrak{B}_{H^{\prime}}(A, B, C, D)$ is the autonomous behavior generated by the Hamiltonian matrix, as the following result shows.

Proposition 12: Let Assumptions 7, 8 hold. Then $\mathfrak{B}_{H^{\prime}}(A, B, C, D)$ can be represented as the set of $w \in$ $\mathfrak{L}_{2}^{\text {loc }}\left(\mathbb{R}, \mathbb{R}^{\mathrm{w}}\right)$ for which exist $x, z \in \mathfrak{L}_{2}^{\text {loc }}\left(\mathbb{R}, \mathbb{R}^{\mathrm{n}}\right)$ such that

$$
\begin{aligned}
{\left[\begin{array}{c}
\dot{x} \\
\dot{z}
\end{array}\right] } & =\left[\begin{array}{cc}
A & B^{\top} \Sigma^{-1} B \\
C^{\top} J C & -A^{\top}
\end{array}\right]\left[\begin{array}{l}
x \\
z
\end{array}\right] \\
w & =\left[\begin{array}{ll}
-\Sigma^{-1} D^{\top} J C & \Sigma^{-1} B^{\top}
\end{array}\right]\left[\begin{array}{l}
x \\
z
\end{array}\right] .
\end{aligned}
$$

Proposition 12 points to how one can compute the antistable part of the set stationary trajectories.

Proposition 13: Let $\mathfrak{B} \in \mathfrak{L}_{\text {contr }}^{\mathrm{w}}, \Sigma=\Sigma^{\top} \in \mathbb{R}^{\mathrm{w} \times \mathrm{w}}$ be nonsingular and $J=\operatorname{block} \operatorname{diag}\left(I_{\text {row }(\mathrm{D})-\mathrm{q}},-I_{\mathrm{q}}\right)$, where $\mathrm{q}$ is number of negative eigenvalues of $\Sigma$. Let $\mathfrak{B}_{O N}(A, B, C, D)$ be a output nulling representation of $\mathfrak{B}$ satisfying assumptions $5,6,7,8$. Then

1. $\mathfrak{B}^{*}$ is the external behavior of $\mathfrak{B}_{H^{\prime}}(A, B, C, D)$, given in (9).

2. $\left[\mathfrak{B}^{*}\right]_{\text {antistable }}=\operatorname{span}\left\{\Sigma^{-1} D^{\top} J C e^{\Lambda_{u} t} X_{1}+\right.$ $\left.\Sigma^{-1} B^{\top} e^{\Lambda_{u} t} Y_{1}\right\}$, where $X_{1} \in \mathbb{R}^{\mathrm{n} \times \mathrm{n}}, Y_{1} \in \mathbb{R}^{\mathrm{n} \times \mathrm{n}}$ are such that $\operatorname{im}\left(\left[\begin{array}{c}X_{1} \\ Y_{1}\end{array}\right]\right)$ satisfies

$$
\left[\begin{array}{cc}
A & B^{\top} \Sigma^{-1} B \\
C^{\top} J C & -A^{\top}
\end{array}\right]\left[\begin{array}{c}
X_{1} \\
Y_{1}
\end{array}\right]=\left[\begin{array}{c}
X_{1} \\
Y_{1}
\end{array}\right] \Lambda_{u},
$$

with $\sigma\left(\Lambda_{u}\right)=\left\{\lambda_{1}, \ldots, \lambda_{\mathrm{n}}\right\}, \lambda_{i} \in \sigma\left(H^{\prime}\right) \bigcap \mathbb{C}_{+}, i=$ $1, \ldots, \mathrm{n}$.

3. For a given $\mathfrak{B}^{\prime} \subset\left[\mathfrak{B}^{*}\right]_{\text {antistable }}$ such that $\mathrm{n}\left(\mathfrak{B}^{\prime}\right)=\mathrm{k}$, there exists a permutation matrix $\Pi$ such that $X_{1} \Pi=$ $\left[\begin{array}{ll}X_{1}^{1} & X_{1}^{2}\end{array}\right], Y_{1} \Pi=\left[\begin{array}{ll}Y_{1}^{1} & Y_{1}^{2}\end{array}\right]$ where $X_{1}^{1}$ and $Y_{1}^{1}$ have $\mathrm{k}$ columns, such that

$$
\begin{aligned}
& {\left[\begin{array}{cc}
A & B^{\top} \Sigma^{-1} B \\
C^{\top} J C & -A^{\top}
\end{array}\right]\left[\begin{array}{ll}
X_{1}^{1} & X_{1}^{2} \\
Y_{1}^{1} & Y_{1}^{2}
\end{array}\right]} \\
& =\left[\begin{array}{ll}
X_{1}^{1} & X_{1}^{2} \\
Y_{1}^{1} & Y_{1}^{2}
\end{array}\right]\left[\begin{array}{cc}
\Lambda_{11} & \Lambda_{12} \\
0 & \Lambda_{22}
\end{array}\right],
\end{aligned}
$$

and

$$
\begin{aligned}
& \mathfrak{B}^{\prime}=\operatorname{span}\left\{\Sigma^{-1} D^{\top} J C e^{\Lambda_{11} t} X_{1}^{1}+\Sigma^{-1} B^{\top} e^{\Lambda_{11} t} Y_{1}^{1}\right\} \\
& \text { with } \Lambda_{u}=\left[\begin{array}{cc}
\Lambda_{11} & \Lambda_{12} \\
0 & \Lambda_{22}
\end{array}\right], \sigma\left(\Lambda_{u}\right)=\left\{\lambda_{1}, \ldots, \lambda_{\mathrm{n}}\right\} \\
& \text { where } \lambda_{i} \in \sigma\left(H^{\prime}\right) \bigcap \mathbb{C}_{+}, i=1, \ldots, \mathrm{n} .
\end{aligned}
$$




\section{MODEL REDUCTION}

We can now describe the algorithms for solving the problem stated in section III.

\section{A. From $\mathfrak{B}$ to reduced-order DV representation}

\section{ALGORITHM 1.}

Input: $\mathfrak{B} \in \mathfrak{L}_{\text {contr }}^{\mathrm{w}}$ strictly $\Sigma$-dissipative on $\mathbb{R}^{-}$, an integer $0 \leq \mathrm{k} \leq \mathrm{n}(\mathfrak{B})$ and a subbehavior $\mathfrak{B}^{\prime}$ of $\left[\mathfrak{B}^{*}\right]_{\text {antistable }}$.

Output: DV representation of $\hat{\mathfrak{B}} \in \mathfrak{L}_{\text {contr }}^{\mathrm{w}}$ solving Problem 1. Step 1. Represent $\mathfrak{B}$ with a driving variable representation $\mathfrak{B}_{D V}(A, B, C, D)$ satisfying assumptions $1,2,3,4$.

Step 2. Compute $X_{1}=\left[\begin{array}{ll}X_{1}^{1} & X_{1}^{2}\end{array}\right], Y_{1}=\left[\begin{array}{ll}Y_{1}^{1} & Y_{1}^{2}\end{array}\right]$ such that

$$
\begin{aligned}
& {\left[\begin{array}{cc}
A & B B^{\top} \\
C^{\top} \Sigma C & -A^{\top}
\end{array}\right]\left[\begin{array}{cc}
X_{1}^{1} & X_{1}^{2} \\
Y_{1}^{1} & Y_{1}^{2}
\end{array}\right]} \\
& =\left[\begin{array}{cc}
X_{1}^{1} & X_{1}^{2} \\
Y_{1}^{1} & Y_{1}^{2}
\end{array}\right] \underbrace{\left[\begin{array}{cc}
\Lambda_{11} & \Lambda_{12} \\
0 & \Lambda_{22}
\end{array}\right]}_{=: \Lambda_{u}},
\end{aligned}
$$

and

$$
\begin{gathered}
{\left[\mathfrak{B}^{*}\right]_{\text {antistable }}=\operatorname{span}\left\{C e^{\Lambda_{u} t} X_{1}+D B^{\top} e^{\Lambda_{u} t} Y_{1}\right\},} \\
\mathfrak{B}^{\prime}=\operatorname{span}\left\{C e^{\Lambda_{11} t} X_{1}^{1}+D B^{\top} e^{\Lambda_{11} t} Y_{1}^{1}\right\},
\end{gathered}
$$

where $\sigma\left(\Lambda_{u}\right)=\left\{\lambda_{1}, \ldots, \lambda_{\mathrm{n}}\right\}, \lambda_{i} \in \sigma(H) \bigcap \mathbb{C}_{+}, i=$ $1, \ldots, \mathrm{n}$.

Step 3. Compute the Cholesky factorization $P^{\top} P=X_{1}^{\top} Y_{1}$, (with $P$ is upper triangular matrix).

Comment: The factorization exists, since $\mathfrak{B} \in \mathfrak{L}_{\text {contr }}^{\mathrm{w}}$ is strictly $\Sigma$-dissipative on $\mathbb{R}^{-}$(Proposition 6) and consequently $X_{1}^{\top} Y_{1}$ is symmetric and positive definite.

Step 4. Define $S=X_{1} P^{-1}=Y_{1}^{-\top} P^{\top}$.

Step 5. Compute

$$
(\bar{A}, \bar{B}, \bar{C}, \bar{D})=\left(S^{-1} A S, S^{-1} B, C S, D\right) .
$$

Step 6. Denote the truncation of $(\bar{A}, \bar{B}, \bar{C}, \bar{D})$ to the first $\mathrm{k}$ component of the state with $\left(\bar{A}_{11}, \bar{B}_{1}, \bar{C}_{1}, \bar{D}\right)$. Denote

$$
\mathfrak{B}_{\text {trunc }}:=\mathfrak{B}_{D V}\left(\bar{A}_{11}, \bar{B}_{1}, \bar{C}_{1}, \bar{D}\right)_{\text {ext }}
$$

Step 7. Perform a Kalman decomposition to compute the controllable part of $\mathfrak{B}_{\text {trunc }}$ :

$$
\begin{aligned}
& T^{-1} \bar{A}_{11} T=\left[\begin{array}{cc}
\hat{A} & * \\
0 & *
\end{array}\right], T^{-1} \bar{B}_{1}=\left[\begin{array}{c}
\hat{B} \\
0
\end{array}\right], \\
& \bar{C}_{1} T=\left[\begin{array}{ll}
\hat{C} & *
\end{array}\right], \bar{D}=\hat{D} .
\end{aligned}
$$

Step 8 Output

$$
\hat{\mathfrak{B}}:=\left[\mathfrak{B}_{\text {trunc }}\right]_{\text {contr }}=\mathfrak{B}_{D V}(\hat{A}, \hat{B}, \hat{C}, \hat{D})_{\text {ext }} .
$$

We now show that the model $\hat{\mathfrak{B}}$ obtained from Algorithm 1 satisfies requirements 1) - 3) of Problem 1 .

1) Since $\mathfrak{B}_{D V}(\hat{A}, \hat{B}, \hat{C}, \hat{D})$ may not be a minimal representation of $\hat{\mathfrak{B}}, \mathrm{n}(\hat{\mathfrak{B}})$ is less than or equal to the size of the matrix $\hat{A} \in \mathbb{R}^{\mathrm{k} \times \mathrm{k}}$.
2) It is easy to see that $\mathfrak{B}_{D V}(\bar{A}, \bar{B}, \bar{C}, \bar{D})$ is also a driving variable representation of $\mathfrak{B}$. Consider the new Hamiltonian matrix generated by $(\bar{A}, \bar{B}, \bar{C}, \bar{D})$

$$
\bar{H}:=\left[\begin{array}{cc}
\bar{A} & \bar{B} \bar{B}^{\top} \\
\bar{C}^{\top} \Sigma \bar{C} & -\bar{A}^{\top}
\end{array}\right]
$$

and the corresponding Hamiltonian system

$$
\left[\begin{array}{cc}
\bar{A} & \bar{B} \bar{B}^{\top} \\
\bar{C}^{\top} \Sigma \bar{C} & -\bar{A}^{\top}
\end{array}\right]\left[\begin{array}{c}
\bar{X}_{1} \\
\bar{Y}_{1}
\end{array}\right]=\left[\begin{array}{c}
\bar{X}_{1} \\
\bar{Y}_{1}
\end{array}\right] \Lambda_{u} .
$$

After using the transformation matrix $S=X_{1} P^{-1}$ we have

$$
\begin{aligned}
\bar{X}_{1} & =S^{-1} X_{1}=\left(P X_{1}^{-1}\right) X_{1}=P, \\
\bar{Y}_{1} & =S^{\top} Y_{1}=\left(P^{-\top} X_{1}^{\top}\right) Y_{1}=P^{-\top} P^{\top} P=P .
\end{aligned}
$$

Hence, the new Hamiltonian system is

$$
\left[\begin{array}{cc}
\bar{A} & \bar{B} \bar{B}^{\top} \\
\bar{C}^{\top} \Sigma \bar{C} & -\bar{A}^{\top}
\end{array}\right]\left[\begin{array}{c}
P \\
P
\end{array}\right]=\left[\begin{array}{c}
P \\
P
\end{array}\right] \Lambda_{u}
$$

Note that since $P$ is an upper triangular matrix, $P=$ $\left[\begin{array}{cc}P_{11} & P_{12} \\ 0 & P_{22}\end{array}\right]$, the Hamiltonian system $(11)$ can be reduced

$$
\left[\begin{array}{cc}
\bar{A}_{11} & \bar{B}_{1} \bar{B}_{1}^{\top} \\
\bar{C}_{1}^{\top} \Sigma \bar{C}_{1} & -\bar{A}_{11}^{\top}
\end{array}\right]\left[\begin{array}{c}
P_{11} \\
P_{11}
\end{array}\right]=\left[\begin{array}{c}
P_{11} \\
P_{11}
\end{array}\right] \Lambda_{11} .
$$

$¿$ From (12) it follows that the largest solution of ARE

$$
\bar{A}_{11}^{\top} \bar{K}+\bar{K} \bar{A}_{11}+\bar{K} \bar{B}_{1} \bar{B}_{1}^{\top} \bar{K}-\bar{C}_{1}^{\top} \Sigma \bar{C}_{1}=0
$$

is $\bar{K}^{+}=P_{11} P_{11}^{-1}=I$. Moreover, from (12) we also have

$$
\left(\bar{A}_{11}+\bar{B}_{1} \bar{B}_{1}^{\top}\right) P_{11}=P_{11} \Lambda_{11} .
$$

This implies that $\sigma\left(\bar{A}_{11}+\bar{B}_{1} \bar{B}_{1}^{\top}\right)$ coincide with $\sigma\left(\Lambda_{11}\right)$ since $P_{11}$ is nonsingular, therefore $\sigma\left(\bar{A}_{11}+\bar{B}_{1} \bar{B}_{1}^{\top}\right) \subset \mathbb{C}^{+}$, hence $\bar{A}_{11}+\bar{B}_{1} \bar{B}_{1}^{\top} I$ is antistable.

Consider the following ARE

$$
\hat{A}^{\top} \hat{K}+\hat{K} \hat{A}+\hat{K} \hat{B} \hat{B}^{\top} \hat{K}-\hat{C}^{\top} \Sigma \hat{C}=0
$$

Since $(\hat{A}, \hat{B}, \hat{C}, \hat{D})$ is obtained from $\left(\bar{A}_{11}, \bar{B}_{1}, \bar{C}_{1}, \bar{D}\right)$ using the Kalman decomposition, it is easy to see that the solution of ARE (14) is the (1,1)-block matrix of the solution of ARE (13). It follows that $I$ is a solution of (14). Moreover, since

$$
\bar{A}_{11}+\bar{B}_{1} \bar{B}_{1}^{\top} I=\left[\begin{array}{cc}
\hat{A}+\hat{B} \hat{B}^{\top} I & * \\
0 & *
\end{array}\right]
$$

it follows that $\hat{A}_{11}+\hat{B}_{1} \hat{B}_{1}^{\top} I$ is antistable. Now use Proposition 6 in order to conclude that $\hat{\mathfrak{B}}$ is strictly $\Sigma$-dissipative on $\mathbb{R}^{-}$.

3) It follows from Proposition 9 that

$$
\hat{\mathfrak{B}}^{*}=\left[\left[\mathfrak{B}_{\text {trunc }}\right]_{\text {contr }}\right]^{*} \subseteq \mathfrak{B}_{H}\left(\bar{A}_{11}, \bar{B}_{1}, \bar{C}_{1}, \bar{D}\right) .
$$

Now note that since $\bar{D}^{\top} \Sigma \bar{D}=I$ and $\bar{D}^{\top} \Sigma \bar{C}_{1}=0$, the conditions of Proposition 7 are satisfied. Consequently

$$
\begin{aligned}
{\left[\hat{\mathfrak{B}}^{*}\right]_{\text {antistable }} } & \subseteq\left[\mathfrak{B}_{H}\left(\bar{A}_{11}, \bar{B}_{1}, \bar{C}_{1}, \bar{D}\right)\right]_{\text {antistable }} \\
& =\operatorname{span}\left\{\left[\bar{C}_{1}+\bar{D} \bar{B}_{1}^{\top}\right] P_{11} e^{\Lambda_{11} t}\right\} \\
& =\mathfrak{B}^{\prime} .
\end{aligned}
$$

Hence, $\left[\hat{\mathfrak{B}}^{*}\right]_{\text {antistable }} \subseteq \mathfrak{B}^{\prime}$. This proves item 3 , and concludes our proof about the correctness of the algorithm. 


\section{B. From $\mathfrak{B}$ to reduced-order ON representation}

\section{ALGORITHM 2.}

Input: $\mathfrak{B} \in \mathfrak{L}_{\text {contr }}^{\mathrm{w}}$ strictly $\Sigma$-dissipative on $\mathbb{R}^{-}$, an integer $0 \leq \mathrm{k} \leq \mathrm{n}(\mathfrak{B})$ and a subbehavior $\mathfrak{B}^{\prime}$ of $\left[\mathfrak{B}^{*}\right]_{\text {antistable }}$.

Output: ON representation of $\hat{\mathfrak{B}} \in \mathfrak{L}_{\text {contr }}^{\mathrm{w}}$ solving Problem 1 . Step 1. Represent $\mathfrak{B}$ by a output nulling representation $\mathfrak{B}_{O N}(A, B, C, D)$ satisfying assumptions $5,6,7,8$.

Step 2. Compute $X_{1}=\left[\begin{array}{ll}X_{1}^{1} & X_{1}^{2}\end{array}\right], Y_{1}=\left[\begin{array}{ll}Y_{1}^{1} & Y_{1}^{2}\end{array}\right]$ such that

$$
\begin{aligned}
& {\left[\begin{array}{cc}
A & B^{\top} \Sigma^{-1} B \\
C^{\top} J C & -A^{\top}
\end{array}\right]\left[\begin{array}{ll}
X_{1}^{1} & X_{1}^{2} \\
Y_{1}^{1} & Y_{1}^{2}
\end{array}\right]} \\
& =\left[\begin{array}{cc}
X_{1}^{1} & X_{1}^{2} \\
Y_{1}^{1} & Y_{1}^{2}
\end{array}\right] \underbrace{\left[\begin{array}{cc}
\Lambda_{11} & \Lambda_{12} \\
0 & \Lambda_{22}
\end{array}\right]}_{=: \Lambda_{u}},
\end{aligned}
$$

and

$$
\begin{gathered}
{\left[\mathfrak{B}^{*}\right]_{\text {antistable }}=\operatorname{span}\left\{\Sigma^{-1} D^{\top} J C e^{\Lambda_{u} t} X_{1}+\Sigma^{-1} B^{\top} e^{\Lambda_{u} t} Y_{1}\right\},} \\
\mathfrak{B}^{\prime}=\operatorname{span}\left\{\Sigma^{-1} D^{\top} J C e^{\Lambda_{11} t} X_{1}^{1}+\Sigma^{-1} B^{\top} e^{\Lambda_{11} t} Y_{1}^{1}\right\},
\end{gathered}
$$

where $\sigma\left(\Lambda_{u}\right)=\left\{\lambda_{1}, \ldots, \lambda_{\mathrm{n}}\right\}, \lambda_{i} \in \sigma\left(H^{\prime}\right) \bigcap \mathbb{C}_{+}, i=$ $1, \ldots, \mathrm{n}$.

Step 3. Compute the Cholesky factorization $P^{\top} P=X_{1}^{\top} Y_{1}$, (with $P$ an upper triangular matrix).

Comment: The factorization exists, since $\mathfrak{B} \in \mathfrak{L}_{\text {contr }}^{\mathrm{w}}$ is strictly $\Sigma$-dissipative on $\mathbb{R}^{-}$(Proposition 6) and consequently $X_{1}^{\top} Y_{1}$ is symmetric and positive definite.

Step 4. Compute $S=X_{1} P^{-1}=Y_{1}^{-\top} P^{\top}$.

Step 5. Compute

$$
(\bar{A}, \bar{B}, \bar{C}, \bar{D}):=\left(S^{-1} A S, S^{-1} B, C S, D\right) .
$$

Step 6. Let $\left(\bar{A}_{11}, \bar{B}_{1}, \bar{C}_{1}, \bar{D}\right)$ denote the truncation of $(\bar{A}, \bar{B}, \bar{C}, \bar{D})$ to the first $\mathrm{k}$ components of the state, and let

$$
\mathfrak{B}_{\text {trunc }}:=\mathfrak{B}_{O N}\left(\bar{A}_{11}, \bar{B}_{1}, \bar{C}_{1}, \bar{D}\right)_{\text {ext }}
$$

Step 7. Find an output injection transformation $H$ to compute the controllable part of $\mathfrak{B}_{\text {trunc }}$ :

$$
\begin{aligned}
& \bar{A}_{11}+H \bar{C}_{1}=\left[\begin{array}{cc}
\hat{A} & * \\
0 & *
\end{array}\right], \bar{B}_{1}+H \bar{D}=\left[\begin{array}{c}
\hat{B} \\
0
\end{array}\right], \\
& \bar{C}_{1}=\left[\begin{array}{ll}
\hat{C} & *
\end{array}\right], \bar{D}=\hat{D} .
\end{aligned}
$$

where $(\hat{A}+F \hat{C}, \hat{B}+F \hat{D})$ is controllable for all real matrices $F$.

Step 8. Output

$$
\hat{\mathfrak{B}}:=\left[\mathfrak{B}_{\text {trunc }}\right]_{\text {contr }}=\mathfrak{B}_{O N}(\hat{A}, \hat{B}, \hat{C}, \hat{D})_{\text {ext }} .
$$

The proof of the correctness of Algorithm 2 follows an argument analogous to that used in proving the correctness of Algorithm 1, and is omitted.

\section{CONCLUSIONS}

The main results of this paper are Algorithms 1 and 2 for the computation of a driving-variable or output-nulling representation of a reduced-order controllable behavior containing a specified subset of the set of stationary trajectories of a given system.
We envision these two algorithms as part of a general scheme for dissipativity-preserving model reduction which, starting from a controllable and dissipative behavior $\mathfrak{B}$ represented in DV, ON, state-space, kernel- or image form, produces any of these representations for a controllable and dissipative reduced-order behavior whose set of stationary trajectories contains a specified subset of the set of stationary trajectories of the original system. Research is being carried out in order to compute a kernel- or image representation of the reduced-order model.

\section{REFERENCES}

[1] A.C. Antoulas, "A new result on passivity-preserving model reduction", System and Control Letters, vol. 54, pp. 361-374, 2005.

[2] Ha Binh Minh, P. Rapisarda, and Trentelman, H.L., "Model Reduction by Retention of Stationary Trajectories", Proceeding of the 17th International Symposium on Mathematical Theory of Networks and Systems, Kyoto, Japan, pp. 1639-1642, July 24-28, 2006.

[3] Madhu N. Belur, Control in a Behavioral Context, Ph.D. Thesis, Rijksuniversiteit Groningen, 2003.

[4] G. Meinsma, Frequency Domain Methods in $H_{\infty}$ Control, Ph.D. Thesis, University of Twente, 1993.

[5] P. Rapisarda, Linear differential systems, Ph.D. Thesis, Rijksuniversiteit Groningen, 1998

[6] P. Rapisarda and H.L. Trentelman, "Linear Hamiltonian behaviors and bilinear differential forms", SIAM J. Contr. Opt., vol. 43, no. 3, pp. 769-791, 2004

[7] D.C. Sorensen, "Passivity preserving model reduction via interpolation of spectral zeros", Systems and Control Letters, vol. 54, pp. 347-360, 2005.

[8] Siep Weiland, Theory of Approximation and Disturbance Attenuation for Linear System, Ph.D. Thesis, Rijksuniversiteit Groningen, 1991.

[9] J.C. Willems and M.E. Valcher, "Linear-quadratic control and quadratic differential forms" IFAC World Congress, Prague, paper MoA15-TO/1, 2005.

[10] Willems, J.C. and Trentelman, H.L., "The dissipation inequality and the algebraic Riccati equation", The Riccati Equation, Springer Verlag, 1991.

[11] Willems, J.C. and Trentelman, H.L., "On quadratic differential forms", SIAM J. Control Opt., vol. 36, no. 5, pp. 1703-1749, 1998.

[12] J.C. Willems and H.L. Trentelman, "Synthesis of dissipative systems using quadratic differential forms - part I", IEEE Transactions on Automatic Control, vol. 47, nr. 1, pp. 53 - 69, 2002. 